\title{
Difference in Neutropenia due to Administration Schedule of TAS-102
}

\author{
Yoichiro Yoshida $^{a}$ Naoya Aisu ${ }^{a}$ Ai Mogi ${ }^{b}$ Akira Komono $^{a}$ \\ Ryohei Sakamoto $^{a}$ Daibo Kojima ${ }^{a}$ Toshiyuki Mera ${ }^{a}$ Suguru Hasegawa ${ }^{a}$ \\ ${ }^{a}$ Department of Gastroenterological Surgery, Fukuoka University Faculty of Medicine, \\ Fukuoka, Japan; ${ }^{b}$ Division of Oncology, Hematology and Infectious Diseases, Department \\ of Internal Medicine, Fukuoka University Faculty of Medicine, Fukuoka, Japan
}

\section{Keywords}

Colorectal cancer $\cdot$ Chemotherapy $\cdot$ TAS-102 $\cdot$ Neutropenia

\begin{abstract}
TAS-102 significantly improves overall survival in patients with metastatic colorectal cancer. The most common adverse event of TAS-102 is bone marrow suppression, which leads to neutropenia. The incidence of neutropenia is high, and there is no known effective prevention method. Furthermore, the administration method of TAS-102 is complicated. We reported that neutropenia could be avoided by changing to a simple administration method of TAS-102.

(C) 2017 The Author(s)

Published by S. Karger AG, Basel
\end{abstract}

\section{Introduction}

TAS-102 is an oral anticancer drug containing trifluridine and tipiracil hydrochloride [1]. Trifluridine is incorporated into DNA, and this process might result in its anticancer effects. Tipiracil hydrochloride assists in maintaining the blood concentrations of trifluridine

Fukuoka University School of Medicine

7-45-1 Nanakuma, Jonan-ku, Fukuoka 814-0180 (Japan)

E-Mail yy4160@yahoo.co.jp 


\section{Case Reports in Oncology}

Case Rep Oncol 2017;10:226-229

DOI: $10.1159 / 000460242$

(C) 2017 The Author(s). Published by S. Karger AG, Base www.karger.com/cro

Yoshida et al.: Difference in Neutropenia due to Administration Schedule of TAS-102

by inhibiting the thymidine phosphorylase that degrades trifluridine. In the global phase III RECOURSE trial, patients who were given TAS-102 demonstrated a significant improvement in overall survival (OS) and progression-free survival (PFS) compared with those given a placebo (hazard ratio, 0.68 and 0.48 for OS and PFS, respectively; both $p<0.0001$ ) [2]. Grade 3 or higher adverse events frequently observed in the TAS-102 arm included neutropenia (38\%) [2]. Neutropenia is an important adverse event that affects the continuation of chemotherapy. Therefore, overcoming neutropenia may be considered to lead to improved survival. In this report, we present a case in which neutropenia could be avoided by changing to a simple administration method of TAS-102.

\section{Case Report}

A 62-year-old woman was referred to our hospital with stage IV descending colon cancer. Colonoscopy revealed a nearly obstructing tumor in the descending colon. Abdominal CT revealed multiple hepatic metastases. The patient underwent a left hemicolectomy (open surgery). KRAS codon 12 showed TGT mutation. We started XELOX + bevacizumab therapy (BV $[7.5 \mathrm{mg} / \mathrm{kg}]$ and oxaliplatin $\left[130 \mathrm{mg} / \mathrm{m}^{2}\right]$ on day 1 plus capecitabine $\left[1,000 \mathrm{mg} / \mathrm{m}^{2}\right]$ twice daily on days $1-14$, every 3 weeks) as a first-line treatment [3] and then started IRIS + bevacizumab therapy (BV $[7.5 \mathrm{mg} / \mathrm{kg}]$ and irinotecan $\left[150 \mathrm{mg} / \mathrm{m}^{2}\right]$ on day 1 plus $\mathrm{S}-1[80$ $\mathrm{mg} / \mathrm{m}^{2}$ ] twice daily on days $1-14$, every 3 weeks) as a second-line treatment [4]. Prior to chemotherapy, the renal, hepatic, and bone marrow functions were almost normal. TAS-102 $\left(35 \mathrm{mg} / \mathrm{m}^{2}\right)$ was administered as a third-line treatment twice daily, after breakfast and dinner, for 5 days a week with 2 days of rest for 2 weeks, followed by a 14-day rest period (Fig. $1 \mathrm{a})$. The results of routine blood analysis after 1 cycle revealed that the white blood cell (WBC) count was 1,200/ $\mu \mathrm{L}$ (normal range, 3,300-8,600/ $\mu \mathrm{L}$ ) and the absolute neutrophil count was $330 / \mu \mathrm{L}$ (normal range, $50-70 \%$ of WBC). Chemotherapy was restarted as neutrophils normalized after 2 weeks' withdrawal. The dose interval was modified without changing the dose intensity. TAS $-102\left(35 \mathrm{mg} / \mathrm{m}^{2}\right)$ was administered twice daily, after breakfast and dinner, for 5 days a week with 9 days of rest for 2 weeks (Fig. 1b). The analysis after the second cycle revealed that the WBC count was $3,100 / \mu \mathrm{L}$ and the absolute neutrophil count was $2,083 / \mu \mathrm{L}$ (Table 1 ). The analysis after the third cycle revealed that the WBC count was $2,500 / \mu \mathrm{L}$ and the absolute neutrophil count was $1,415 / \mu \mathrm{L}$.

\section{Discussion}

Neutropenia is the most common cause of chemotherapy dose reduction and delay [5]. The frequency of neutropenia differs according to the chemotherapy regimen even if the same anticancer drug is administered [6, 7].Grade 3-4 hematologic toxicity, mainly neutropenia, was approximately seven times more frequent in the 5-FU bolus arm, whereas handfoot syndrome was approximately two times more frequent in the 5-FU continuous infusion arm [8]. Prolonged infusion may expose a relatively larger proportion of tumor cells to 5-FU [6]. Moreover, extending the time of infusion is an attractive option for tumors with a relatively slow doubling time, such as colorectal cancers $[9,10]$. It has been suggested that the 
mechanisms of resistance to 5-FU depend on the modality of its administration. Bolus administration of 5-FU inhibits RNA synthesis, whereas continuous infusion may be more cytotoxic via the inhibition of thymidilate synthase [11]. Delivering 5-FU by continuous infusion rather than by bolus intravenous infusion permits the delivery of higher drug doses and changes the limiting toxicity from myelosuppression to stomatitis and hand-foot syndrome. Examining the findings revealed that adverse events also differ when administration methods are different. Although details on TAS-102 are unknown, our outcome suggests that neutropenia caused by TAS-102 may be associated with the dosing interval.

Patients who develop neutropenia experience an increased risk of serious infections and often require hospitalization [12]. Therefore, the prevention and management of neutropenia is an important issue in chemotherapy of CRC. A modified regimen may reduce the risk of infection and hospitalization. However, since the anticancer effect is unknown, further study is necessary.

To our best knowledge, this is the first report in which neutropenia could be prevented by changing to a simple administration method of TAS-102. The work is original. However, given the low number of patients considered, it is not possible to come to any well-defined conclusion. Based on the present case, several changes may be necessary, including how best to individualize dosing schedules.

\section{Statement of Ethics}

The patient gave written informed consent.

\section{Disclosure Statement}

The authors declare that they have no conflicts of interest to disclose.

\section{References}

1 Emura T, et al: A novel antimetabolite, TAS-102 retains its effect on FU-related resistant cancer cells. Int J Mol Med 2004;13:545-549.

-2 Mayer RJ, et al: Randomized trial of TAS-102 for refractory metastatic colorectal cancer. N Engl J Med 2015;372:1909-1919.

-3 Yoshida Y, et al: Administration of chemotherapy via the median cubital vein without implantable central venous access ports: port-free chemotherapy for metastatic colorectal cancer patients. Int J Clin Oncol 2015;20:332-337.

-4 Yamada Y, et al: Phase II study of oral S-1 with irinotecan and bevacizumab (SIRB) as first-line therapy for patients with metastatic colorectal cancer. Invest New Drugs 2012;30:1690-1696.

5 Yoshida Y, et al: Can grade 2 neutropenia predict the risk of grade 3 neutropenia in metastatic colorectal cancer patients treated with chemotherapy? Support Care Cancer 2015;23:1623-1627.

6 Piedbois P, et al: Efficacy of intravenous continuous infusion of fluorouracil compared with bolus administration in advanced colorectal cancer. J Clin Oncol 1998;16:301-308.

7 Yoshida Y, et al: Clinical significance of bolus 5-fluorouracil for recurrent or metastatic colorectal cancer treated with FOLFOX + bevacizumab therapy (in Japanese). Gan To Kagaku Ryoho 2011;38:1293-1296. 
8 Levy E, et al: Toxicity of fluorouracil in patients with advanced colorectal cancer: effect of administration schedule and prognostic factors. J Clin Oncol 1998;16:3537-3541.

9 Myers CE, et al: Pharmacokinetics of the fluoropyrimidines: implications for their clinical use. Cancer Treat Rev 1976;3:175-183.

10 Fraile RJ, et al: Pharmacokinetics of 5-fluorouracil administered orally, by rapid intravenous and by slow infusion. Cancer Res 1980;40:2223-2228.

$\checkmark 11$ Aschele C, et al: Novel mechanism(s) of resistance to 5-fluorouracil in human colon cancer (HCT-8) sublines following exposure to two different clinically relevant dose schedules. Cancer Res 1992;52:1855-1864.

12 Ho MY, Mackey JR: Presentation and management of docetaxel-related adverse effects in patients with breast cancer. Cancer Manag Res 2014;6:253-259.

a

\begin{tabular}{|l|l|l|l|l|l|}
\hline TAS-102 & & TAS-102 & & \\
\hline 5 days & 2 days & 5 days 2 days & & \\
& & & \\
\hline
\end{tabular}

1 cycle $=28$ days

Fig. 1. TAS-102 regimen. a Regular regimen. b Modified regimen.

Table 1. White blood cells (WBC) and neutrophils $(/ \mu \mathrm{L})$ after chemotherapy

\begin{tabular}{llcc}
\hline & Regimen & WBC & Neutrophil \\
\hline 1st cycle & A & 1,200 & 330 \\
2nd cycle & B & 3,100 & 2,083 \\
3rd cycle & B & 2,500 & 1,415 \\
\hline
\end{tabular}

